\title{
Erratum to: Genotype distribution-based inference of collective effects in genome- wide association studies: insights to age- related macular degeneration disease mechanism
}

Hyung Jun Woo', Chenggang Yu' ${ }^{1}$, Kamal Kumar ${ }^{1}$, Bert Gold² and Jaques Reifman ${ }^{1 *}$

\section{Erratum}

During the publication process, the published version of the original article [1] had a duplicate image of Additional File 3 in place of Fig. 2. The graphics for Fig. 2 have now been restored in the original article [1].

\begin{abstract}
Author details
'Biotechnology High Performance Computing Software Applications Institute, Telemedicine and Advanced Technology Research Center, U.S. Army Medical Research and Materiel Command, Fort Detrick, Maryland, USA. 2Laboratory of Genomic Diversity, National Cancer Institute, Frederick, Maryland, USA.
\end{abstract}

Received: 30 August 2016 Accepted: 15 September 2016 Published online: 22 September 2016

\section{Reference}

1. Woo, et al. Genotype distribution-based inference of collective effects in

genome-wide association studies: insights to age-related macular degeneration disease mechanism. BMC Genomics. 2016;17:695. doi:10.1186/s12864-016-2871-3.

* Correspondence: jaques.reifman.civ@mail.mil 


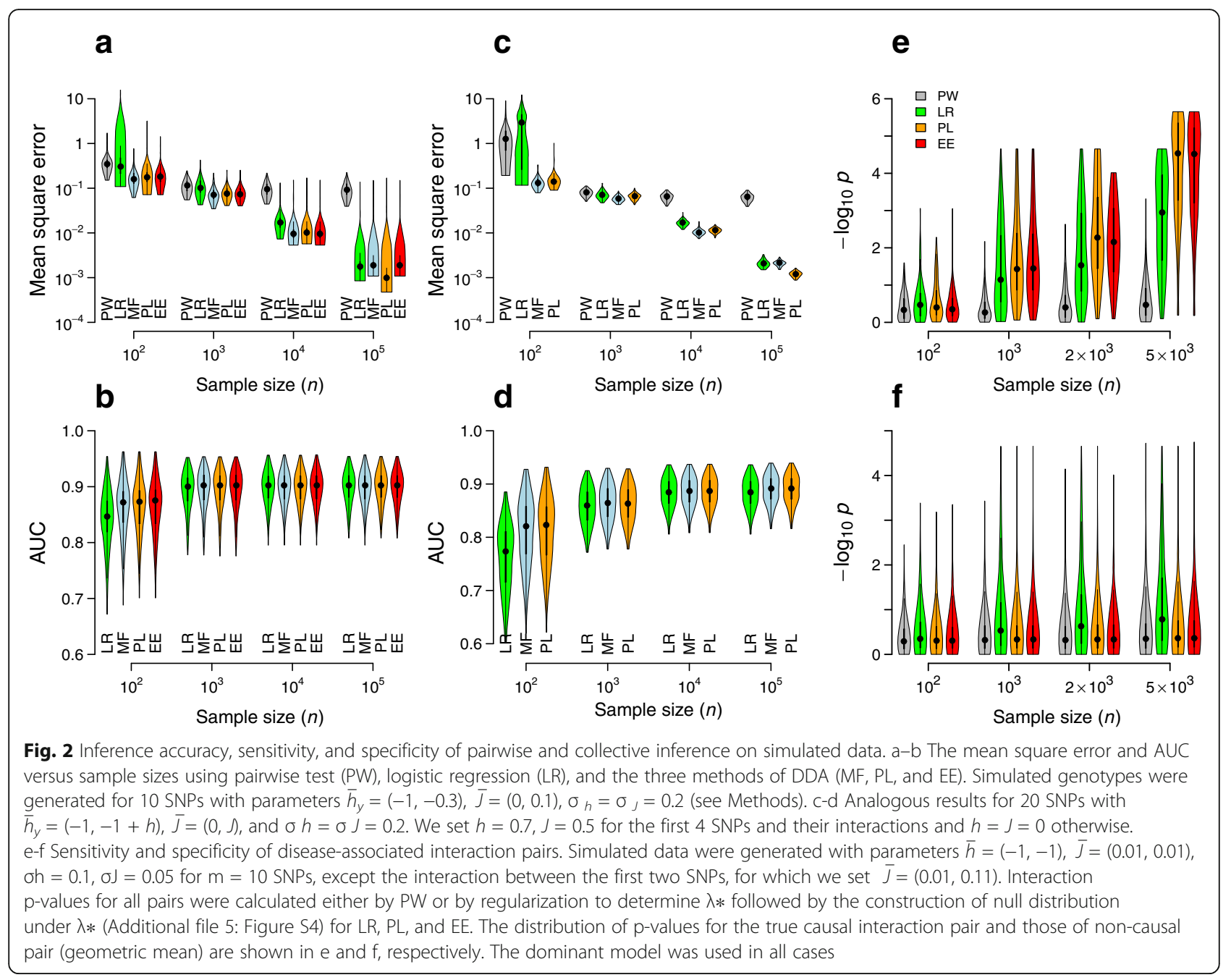

